\title{
Micro-LED arrays for spatio-temporally correlated multi-mode operation
}

\author{
Johannes Herrnsdorf, Alexander D. Griffiths, Enyuan Xie, Jonathan McKendry, Erdan Gu, Michael J. Strain, and Martin D. Dawson \\ ${ }^{1}$ Institute of Photonics, SUPA, Department of Physics, University of Strathlcyde, Glasgow G1 1RD, United Kingdom \\ m.dawson@strath.ac.uk
}

\begin{abstract}
Micro-LEDs are the basis of attractive new forms of high brightness and high resolution displays. We discuss how broader conceptions and convergences with lighting, communications, imaging and sensing are emerging from this rapidly developing technology.
\end{abstract}

Keywords-micro-LED, electronic display, visible light communications, structured illumination

\section{INTRODUCTION}

Micro-LED technology, based principally on the capabilities of gallium nitride inorganic semiconductor epistructures, is emerging very rapidly to commercial maturity to provide a new generation of high-performance displays [1]. These devices, which comprise high-density arrays of individual LED pixels with dimensions of a few microns to several tens of microns, are of the self-illumination variety with high-brightness [2], broad viewing angle, and very rapid (ns) response time [3]. Advanced demonstrators spanning wearables, to augmented reality (AR) and mixed reality (MR) systems, through to large screen TVs and displays, are currently being trialed by research laboratories, innovative new businesses and multi-national corporations. Significantly, these demonstrator display panels include those of a scale and brightness suitable, where appropriate, for combining lighting and display functions.

In parallel with these developments, the lighting industry - in its move to solid-state lighting - is beginning to embrace new operating models (such as Lighting-as-a-Service) which will combine lighting with novel functionality such as optical wireless communications via LiFi (Light Fidelity). The developments underway promise advances from firstgeneration, so-called 'smart lighting' towards what might be called digital lighting under sophisticated electronic control. In addition to its role in displays, micro-LED technology is also setting performance benchmarks in LiFi [3], and the exciting prospect is emerging of combining micro-LED based capabilities for displays with lighting and communications, potentially also involving other functions including sensing, ranging and imaging. Micro-LED technology is therefore expected to help facilitate convergences in these hitherto largely separate areas of technological application.

\section{MICRO-LEDS IN DISPLAYS, LIGHTING AND COMMUNCIATIONS}

Micro-LEDs naturally facilitate high-pixel-density 1-D and 2-D emitter array formats to provide electronic visual display capability. Depending upon the specific type of display, this can span pixels down to $\sim 1 \mu \mathrm{m}$ in dimension/diameter on a few-micron pitch, to pixels up to around $50 \mu \mathrm{m}$ in dimension/diameter on a pitch of 100 's $\mu \mathrm{m}$ to $\sim \mathrm{mm}$. Gallium nitride technology readily provides blue and green emitter material for these devices and, where required, more specialised wavelengths from the deep ultraviolet though to amber. For red emission, several approaches are being explored, but the most established include use of colloidal quantum dot color converters (optically excited by longer wavelength nitride micro-pixels) or AlInGaP/GaAs semiconductors in micro-pixel format. The detailed physics of micro-sized LED pixels offers enhanced modulation bandwidths into the $\mathrm{GHz}$ range per pixel, two orders of magnitude higher than is typical for conventional broad area $\left(0.1-1 \mathrm{~mm}^{2}\right.$ per chip) GaN LEDs [3]. These capabilities can be combined in new forms of spatially multiplexed or spatially modulated visible light communications, such as space-shift keying (SSK) or multiple input multiple output (MIMO), to enhance data communications channel capacity [3]. In this format, the display function embodies the spatial registration/distribution of information in a communications link, rather than necessarily embodying direct view physically meaningful images. However, the frame rate or image refresh rate is so fast for micro-LEDs (potentially $>1 \mathrm{MHz}$ ) that the displays could operate multi-modally, to implement display and spatially modulated optical wireless communications functions in parallel.

Furthermore, high refresh $(\mathrm{MHz})$ rate binary mask patterns can be generated with micro-LED displays, potentially either in monochrome or sequential color. These systems can project such checkerboard-like high-frame-rate patterns to provide unique digital signatures to each location in the imaged frame, which can be used to implement location, tracking and navigation functions [4]. Thus the projected output of the display can be used e.g. to locate and track moving objects in the space around it, potentially including viewers of the display.

\section{SUMMARY}

Micro-LED technology brings the full power of sophisticated electronic interfacing and communications technology into the arena of displays and lighting, and facilitates the interaction of displays with their environment. The presentation will review these exciting emerging capabilities and speculate on how they may develop.

\section{ACKNOWLEDGMENT}

EPSRC support for micro-LEDs since 2001, most recently grants EP/K00042X/1, EP/M01326X/1, and EP/P02744X/2

\section{REFERENCES}

J. Day et al., "III-Nitride full-scale high-resolutionj microdisplays," Appl. Phys. Lett., vol. 99, 031116, 2011.

[2] J. Herrnsdorf et al., "Active matrix GaN micro-LED display with unprecedented brightness," IEEE Trans. Electron. Dev., vol. 62, no. 6, pp. 1918-1925, 2015.

[3] S. Rajbhandari et al., "A review of galium nitride LEDs for multi$\mathrm{Gb} / \mathrm{s}$ visible light data communications," Semicond. Sci. Technol., Vol. 32, 023001, 2017.

[4] J. Herrnsdorf et al., "Positioing and data broadcasting using illumination pattern sequebces displayed by LED arrays," IEEE Trans. Comm., vol. 66, pp 5582-5592, 2018. 
